\title{
Research on the Traffic Flow Control of Urban Occasional Congestion Based on Catastrophe Theory
}

\author{
Yang Xu $\mathbb{D},{ }^{1}$ Duo Jia Zhang, ${ }^{1}$ Xin Zhang $\mathbb{D},{ }^{1}$ Kin Keung Lai, ${ }^{2}$ and Bing Su $\mathbb{D}^{1}$ \\ ${ }^{1}$ School of Economics \& Management, Xi'an Technological University, Xi'an 710021, China \\ ${ }^{2}$ School of Economics and Commerce, Guangdong University of Technology, Guangzhou, China \\ Correspondence should be addressed to Xin Zhang; zhangxin317424@163.com
}

Received 13 May 2021; Accepted 26 July 2021; Published 3 August 2021

Academic Editor: Elżbieta Macioszek

Copyright $(02021$ Yang Xu et al. This is an open access article distributed under the Creative Commons Attribution License, which permits unrestricted use, distribution, and reproduction in any medium, provided the original work is properly cited.

Aimed at the problem of occasional congestion control, the cusp catastrophe theory is used to establish the catastrophe model of the urban road system under occasional congestion, finding breakpoints and analyzing stability after urban road system catastrophes by constructing the energetic function; based on the catastrophe characteristics of the urban road system, the feasibility method of congestion control is discussed. The results show that the control method of traffic flow based on catastrophe characteristics of the urban road system can effectively improve the efficiency of the road system in theory. Finally, the applicability of the control method based on catastrophe characteristics is analyzed by examples under different occasional congestion situations.

\section{Introduction}

Occasional congestion refers to the phenomenon of traffic congestion caused by the decline of road capacity due to occasional accidents. Occasional congestion often adversely affects the efficiency of the urban road system, and improper control may even cause the congestion to spread to upstream intersections and form regional blockages. Therefore, the research on the control of urban road congestion has important theoretical significance and practical value.

In existing research on the control of occasional congestion, the goal is to minimize the delay time [1], or limit the density [2], or the maximum capacity [3]; the control methods mainly adopt the signal light control [4]. The above research mainly focuses on the single-lane situation. In this paper, for the multilane situation, take the maximum capacity as the goal, the control method of traffic flow based on catastrophe characteristics of urban road system is proposed, and the efficiency of the control method under different occasional congestion situations is discussed.

\section{Catastrophe Theory and the Urban Road System}

Catastrophe theory is the French mathematician Thom's theory of discontinuous change based on mathematical theories such as singularity theory and stability theory [5]. In catastrophe theory, potential refers to the ability of the system to develop in a certain direction. The independent variable of the potential function is the state variable used to describe the behavior of the system, and the factor that affects the change of the behavior of the system is the control variable. Existing studies have summarized 7 primary catastrophes when the number of state variables does not exceed two and the number of control variables does not exceed four: cusp catastrophe, folding catastrophe, dovetail catastrophe, hyperbolic umbilical point catastrophe, butterfly catastrophe, ellipse umbilical point catastrophe, and parabolic umbilical point catastrophe [6]. For a system with a catastrophe phenomenon, as long as the number of its state variables and control variables is established, one of the corresponding seven elementary catastrophe functions can be selected as the catastrophe model of the system to analyze 
the catastrophe characteristics of the system. The basic idea of catastrophe theory is to use bifurcation theory to analyze the stability of the system, to classify breakpoints according to potential functions, to study the characteristics of discontinuous states near various breakpoints, to find the breakpoints of potential function catastrophes, and to analyze the system by the process of catastrophe from one stable state to another stable state [7]. Catastrophe theory has very strong applicability and has been applied to various fields since it was proposed.

Cusp systems generally have five major characteristics: sudden jump, multimodality, unreachability, divergence, and hysteresis [6]. The road traffic system is a complex system described by multiple parameters. Its operation process conforms to the five characteristics of cusp abrupt changes: sudden jump (the system transition from a noncrowded state to a crowded state is not a gradual process, but a leap, a catastrophe), multimodality (the system has two steady states, crowded and noncrowded), unreachability (some areas in the system cannot be reached in practice), bifurcation (when the system is in a critical equilibrium state, where just an unstable balance exists instantaneously. Once it encounters disturbances from external factors, this balance may be destroyed. The system may be biased to a crowded state or a noncrowded state), divergence (in a critical state, small changes in control variables will lead to a catastrophe of the state variable), and hysteresis (the direction of the catastrophe is related to the direction of the control variable, and the catastrophe of the control variable from one direction is different from the catastrophe from the other direction) [8].

This paper uses the cusp catastrophe theory to analyze the catastrophe characteristics of the urban road system under occasional congestion. Taking the occupancy rate of the traffic volume in the carrying capacity as the state variable, and the change rate of the traffic flow and the change rate of the road capacity as the control variables, the cusp catastrophe model of the urban road traffic system under occasional congestion is established. The impact of occasional congestion on the road traffic system is first manifested as changes in control variables, which further cause state variables to change in multiple state spaces and even the system catastrophes. The cusp catastrophe model is used to analyze the catastrophe characteristics of the system, determining breakpoints and analyzing stability after the urban road system catastrophes. Finally, we give a feasible method for traffic flow control.

\section{Catastrophe Model}

3.1. State Variables and Control Variables of the Urban Road System. In this paper, the occupancy rate of traffic volume in the carrying capacity is selected as the state variable of the system. Traffic flow and road capacity are the two control variables of the system, in order to maintain the same dimension as the state variable, the change rate of traffic flow and the change rate of road capacity are selected as the control variable of the system, and the cusp catastrophe model is established to analyze the catastrophe characteristics of the road traffic system under occasional congestion.

Definition 1. The occupancy rate of the traffic volume in the carrying capacity is taken as a state variable, denoted as $\eta(t)$ :

$$
\eta(t)=\frac{X(t)}{Y(t)} \times 100 \%
$$

where $X(t)$ is the traffic volume of the road system and $Y(t)$ is the carrying capacity of the road the system.

Definition 2. The change rate of the traffic flow is regarded as a control variable of the system, denoted as $\gamma(t)$ :

$$
\gamma(t)=\frac{Q(t)-Q(0)}{Q(0)} \times 100 \%
$$

where $Q(0)$ is the traffic flow at the moment of the accident and $Q(t)$ is the traffic flow at the moment $t$.

Definition 3. Take the change rate of the road capacity as another control variable of the system, denoted as $\lambda(t)$ :

$$
\lambda(t)=\frac{C(0)-C(t)}{C(0)} \times 100 \%,
$$

where $C(0)$ is the road capacity at the moment of an accident and $C(t)$ is the capacity of the road at the moment $t$.

In practice, urban roads often use multiple lanes in the same direction in urban planning. When accidents occur, the lanes are occupied and the road capacity will inevitably decrease. Occasional accidents occur in different locations and have different impacts on road capacity. The impact value is mainly determined by the lane utilization coefficient. Take three lanes as an example. From the center line of the road to the rightmost lane, they are defined as lanes 1,2, and 3 respectively. The utilization coefficient of the lane is gradually reduced, and the utilization coefficient of each lane is $1.00,0.8-0.89$ (take 0.87 ), and $0.65-0.78$ (take 0.73 ), respectively; then, when an accident occurs, the road capacity $C$ is

$$
C=C_{0} P \beta_{j}
$$

where $C_{0}$ is the design capacity of the road and $P$ is lane loss coefficient, expressed by the ratio of the number of lanes available to the total number of lanes [9]:

$$
P=\frac{N-n}{N} \times 100 \%,
$$

where $n$ is the number of damaged lanes and $N$ is the number of total lanes.

In equation ( 4$), \beta_{j}$ denotes the weight which is the sum of utilization coefficients of available lanes to total lane utilization coefficients, as occasional congestion occurring in lane $i$ :

$$
\beta_{j}=\frac{\sum \alpha_{i}-\sum \alpha_{i}^{*}}{\sum \alpha_{i}}, \quad i=1,2,3 \text {, }
$$


where $\sum \alpha_{i}^{*}$ is the sum of utilization coefficients of lanes occupied or damaged as occasional congestion occurring in lane $i$ and $\sum \alpha_{i}$ is total lane utilization coefficients.

\subsection{Cusp Catastrophe Model of the Urban Road System under} Occasional Congestion. Taking the occupancy rate of the traffic volume in the carrying capacity as the state variable, and the change rate of traffic flow and the change rate of capacity as the two control variables, the cusp catastrophe model of urban road system under occasional congestion is established, and its potential function is

$$
E(\eta)=\eta(t)^{4}+\gamma(t) \eta^{2}(t)+\lambda(t) \eta(t) .
$$

According to $E^{\prime}(\eta)=0$, the equilibrium surface (catastrophe manifold) is

$$
4 \eta^{3}(t)+2 \gamma(t) \eta(t)+\lambda(t)=0 .
$$

According to $E^{\prime \prime}(\eta)=0$, the singularity is

$$
12 \eta^{2}(t)+2 \gamma(t)=0 .
$$

Combine equations (8) and (9) to obtain the bifurcation set:

$$
8 \gamma(t)^{3}+27 \lambda(t)^{2}=0 .
$$

The urban road system is a three-dimensional space composed of state variables $\eta(t)$ and control variables $\lambda(t)$, $\gamma(t)$. The equilibrium surface is a smooth surface containing folds (or pleats). The upper and lower sheets of the surface are stable equilibrium points. In the middle sheet, the system moves in the direction which minimizes potential function; at this state, the equilibrium point is unstable, so it is called the unreachable region, while in the upper and lower sheets, the potential function reaches the minimum value and the equilibrium point is stable, which is also the state the potential function is usually located in. The bifurcation set is defined by projection of the fold, which determines the area of the catastrophic behavior [10].

When control variables move outside the bifurcation set, the corresponding equilibrium point changes in the upper or lower of the manifold, and the system is stable; when control variables move through the bifurcation set point, the corresponding equilibrium point is at the edge of the upper and lower of the manifold; at this time, the system is about to undergo a catastrophe; when control variables move around the inner region of the bifurcation set, the equilibrium point is at the middle sheet of the manifold, and the system is in an unstable state, and it jumps to another state at any time.

\section{Catastrophe Analysis}

When using the cusp catastrophe model to analyze the catastrophe process of the road traffic system under occasional congestion, it is necessary to construct the energetic function of the system to describe the relationship between the variables. The energetic function is the complex sum term of the potential function, which has the same catastrophe characteristics as the potential function [6].
Therefore, through analyzing the energetic function of the system, breakpoints of the system can be solved and the stability of the system can be discussed. On this basis, the feasibility control method of the flow under the occasional congestion can be obtained. follows:

The energetic function of the road traffic system [3] is as

$$
E(t)=\frac{1}{2} m Q(t) V^{2}(t)
$$

where $m$ is the mass coefficient of the standard unit vehicle and $V(t)$ is the average speed of the vehicle.

4.1. Speed Model Based on the Relationship between Traffic Flow Parameters. Traffic flow $Q$, average speed $V$, and density $K$ are the three basic parameters that characterize traffic flow, and the basic relationship between the three is [11]: $Q=V K$. According to the Greenshields velocitydensity linear model [12], $V=a K+b$, where $a$ and $b$ are the variable coefficients, which can be obtained through data regression analysis. The flow-density relationship is thus obtained as

$$
Q=a K^{2}+b K
$$

Let $(\mathrm{dQ} / \mathrm{d} K)=0$, that is, $2 a K+b=0$, obtaining the following: when $K=-(b / 2 a), V=b / 2$, the flow reaches the maximum value: $Q_{\max }=-\left(b^{2} / 4 a\right)$; the following road capacity $C$ is formulated:

$$
C=-\frac{b^{2}}{4 a}
$$

Let the length of the road be $L$, the time when the vehicle passes in the free flow state is expressed as $T_{0}=L / b$; meanwhile, $V=L / T, K=(V / a)-(b / a)$, and combining with flow-density relationship (12), we obtain

$$
Q=-4 C\left(\frac{T_{0}}{T}\right)^{2}+4 C \frac{T_{0}}{T}
$$

Formula (14) can be regarded as a quadratic equation of one variable about $T_{0} / T$, and the path resistance function can be obtained by solving

$$
T=T_{0}\left(\frac{2}{1 \pm \sqrt{1-(Q / C)}}\right) .
$$

The application range of the road impedance function model is $0 \leq(Q / C) \leq 1$, the degree of road load $Q / C$ as the independent variable, denoting different vehicle flow speeds. When $Q / C=1$, that is, $T / T_{0}=2$, it means that the vehicle travel time is twice the free travel time, when the road is in a saturated state [13].

Based on the independent resistance function, the average speed $V(t)$ of vehicles in the urban road system can be expressed as

$$
V(t)=\frac{b}{[2 / 1+\sqrt{1-(Q(t) / C(t))}]}
$$


4.2. Catastrophe Analysis of the Urban Road System under Occasional Congestion. From equations (11) and (16), the energetic function of the urban road system is

$$
E(K(t), C(t))=\frac{1}{8} m\left(a K^{2}(t)+b K(t)\right) b^{2}\left(1+\sqrt{1-\frac{a K^{2}(t)+b K(t)}{C(t)}}\right)^{2} .
$$

The set of breakpoints of the energetic function can be obtained by $\partial E(K(t), C(t)) / \partial K(t)=0$, which can be simplified as

$$
\left(a K(t)+\frac{1}{2} b\right)\left[1+\sqrt{1-\frac{a K^{2}(t)+b K(t)}{C(t)}}-\frac{a K(t)^{2}+b K(t)}{C(t) \sqrt{1-\left(a K^{2}(t)+b K(t) / C(t)\right)}}\right]=0
$$

and the breakpoints are obtained:

$$
K(t)=\frac{3}{2 b} C(t) .
$$

Whether the second derivative is negative or not is the condition for judging the stability of breakpoints:

$$
\frac{\partial^{2} E(K(t), C(t))}{\partial K(t)^{2}}=\left[2 a K(t)+\frac{3}{2} b\right]\left[2 a K(t)+\frac{1}{2} b\right]+\frac{[a K(t)+1 / 2 b]^{2} a K^{2}(t)+b K(t)}{C(t)\left(1-\left(a K^{2}(t)+b K(t) / C(t)\right)\right)}
$$

simplified as

$$
K(t)<\frac{3}{b} C(t)
$$

When $\partial^{2} E(K(t), C(t)) / \partial K(t)^{2}>0$, the energetic function has a minimum point, breakpoints can be judged to be a stable breakpoint; when $\partial^{2} E(K(t), C(t)) / \partial K(t)^{2}<0$, the energetic function has a maximum value, and breakpoints can be judged to be an unstable breakpoint; when $\partial^{2} E(K(t), C(t)) / \partial K(t)^{2}=0$, it is the inflection point of the energetic function with respect to density.

Therefore, the break flow $Q(i)$ of the urban road system at the catastrophe time can be obtained:

$$
Q(i)=a\left(\frac{3 C(i)}{2 b}\right)^{2}+\frac{3 C(i)}{2} \text {. }
$$

After the catastrophe, the potential function will continue to move continuously to the minimum value until it is stable at time $j$; then the stable traffic flow will be

$$
Q(j)=\frac{9 a}{b^{2}} C^{2}(j)+3 C(j)
$$

Suppose that when an accident occurs at 0 moment, the road capacity becomes smaller and the control variable $\lambda(t)$ of the system becomes larger instantly. According to Maxwell's agreement, the potential function will move to a minimum point, and also $\gamma(t)$ begins to change; at time $i$, the potential function reaches the junction of the bifurcation set point; that is, when the control variable satisfies the bifurcation set point: $8 \gamma(i)^{3}+27 \lambda(i)^{2}=0$, the potential function suddenly becomes smaller, discontinuity occurs, and the system jumps from the original stable state to another stable state; as the control variable continues to change, the potential function continues to move slowly and continuously to the minimum value; the system stabilizes after reaching the equilibrium point $j$ in the new stable area and obtains a stable traffic flow: $Q(j)=\left(9 a / b^{2}\right) C^{2}(j)+3 C(j) \quad$ (see Figure 1).

\section{Traffic Flow Control Method Based on Catastrophe Characteristics of the Urban Road System}

Catastrophe theory has two main catastrophe conventions: ideal delay convention and Maxwell convention. The former believes that the system has been stable at the established equilibrium position and remains unchanged until the equilibrium position disappears; the latter refers to the general direction of the system that makes the overall potential extremely small to shift to the equilibrium position. The road traffic system is affected by the control variables and its operation changes are more random. Therefore, the 


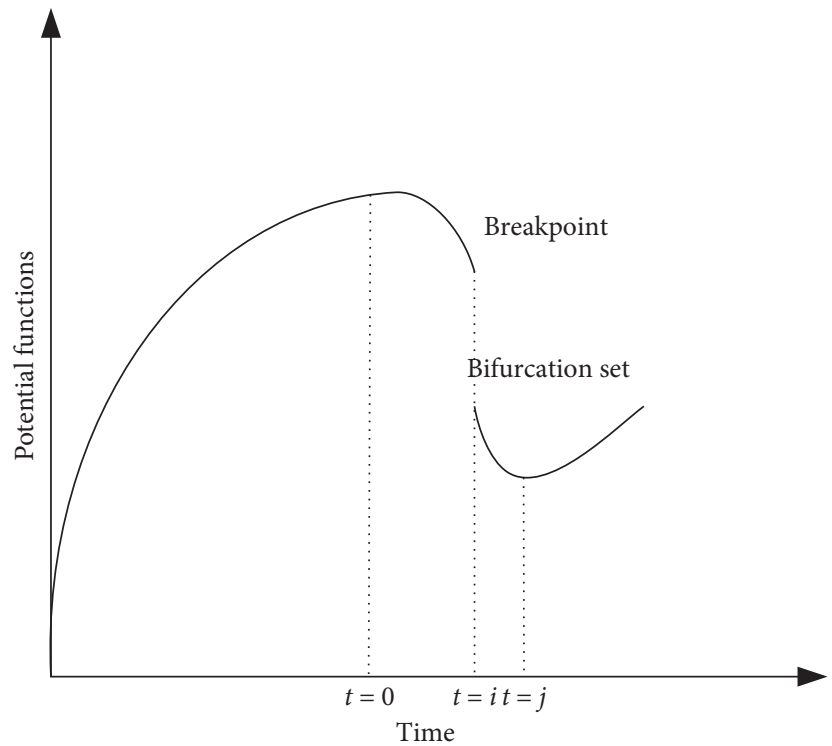

Figure 1: The change graph of the potential function of occasional congestion.

Maxwell agreement is more suitable for the road traffic system.

In the catastrophe model, the larger the potential function, the higher the operating efficiency of the urban road system. According to Maxwell's agreement, the potential function always moves to the smallest equilibrium position of the potential global, from one stable area to another stable area, until the equilibrium is stable in another area; that is, the system has a catastrophe. At this time, the potential function can only move to the maximum direction with the help of external force, that is, adopt the corresponding traffic flow control strategy to improve the operating efficiency of the urban road system.

Based on the analysis of the catastrophe process of urban road system, at a critical point the stable points, and the bifurcation set, the potential function suddenly becomes smaller, and the system suddenly changes from the original stable region to another stable region and continuously becomes smaller in the new stable region until balanced. Based on this, the feasibility control methods of the traffic flow are given: (1) Before a catastrophe, control the traffic flow to make it less than the breakpoints. Preventing the catastrophe occurs, and the potential function decreases sharply. (2) After a catastrophe, control the traffic flow with a certain restriction rate to be as small as the stable traffic flow, preventing the potential function moving to a smaller direction, in order to obtain the maximum capacity. According to formulas (22) and (23), the traffic flow restriction rate is obtained [3]:

$$
\gamma *(t)=\left[1-\frac{4\left(3 a C(i)+b^{2}\right)}{3 a C(i)+2 b^{2}}\right] \times 100 \% .
$$

\section{Experiment}

6.1. Experimental Parameters. In this paper, a cellular automaton model is used to simulate the evolution of a three- lane road traffic flow under occasional accidents. Construct a one-direction and three-lane road system with a length of 800 cells (one cell is $3.75 \mathrm{~m}$ in length equal to $3 \mathrm{~km}$ in actual length). The design capacity of the road system is $4200 \mathrm{puc} / \mathrm{h}$, and the maximum speed of the vehicle $v=67.5 \mathrm{~km} / \mathrm{h}$. The forced lane change probability and random slowdown rate of ordinary vehicles are 0.7 and 0.3 , respectively. The vehicle enters the road with probability of 0.7 from the area which is at the entrance of each lane with a length of 6 cells. Starting from $1000 \mathrm{~s}$ before the accident, data is collected $100 \mathrm{~s}$ per interval until the accident lasts $2000 \mathrm{~s}$, and the traffic flow evolution data with a duration of $3000 \mathrm{~s}$ is obtained (see Table 1).

6.2. Results and Analysis. The parameters describing the urban road system after urban road system catastrophes under the three occasional situations are calculated (see Table 2).

Based on the parameter after urban road system catastrophes, the flow restriction rates under three occasional congestion situations are calculated separately, and a comparison is drawn between the operating efficiency of the urban road system before controlling and after control, obtaining the efficiency of the traffic flow controlling (see Table 3).

The analysis of the example shows that, under occasional congestion, the traffic flow control method based on the catastrophe characteristics of the road system can effectively improve the operation efficiency of the road system and improve the road capacity. The efficiency of traffic flow controlling is related to the location of the accident and the number of lanes occupied. The results show the following: when one lane is occupied, the efficiency of traffic flow controlling is higher than when two lanes are occupied. In the case of a lane being occupied, the efficiency of traffic flow controlling when lane 2 is occupied is lower than when lane 
TABle 1: Traffic flow simulation data under occasional accidents.

\begin{tabular}{|c|c|c|c|c|c|c|c|c|c|}
\hline \multirow[b]{2}{*}{ Number } & \multicolumn{3}{|c|}{ Lane 2 is occupied } & \multicolumn{3}{|c|}{ Lane 3 is occupied } & \multicolumn{3}{|c|}{ Lanes 2 and 3 are occupied } \\
\hline & $\begin{array}{l}\text { Speed, } \\
\mathrm{km} / \mathrm{h}\end{array}$ & $\begin{array}{l}\text { Density, } \\
\text { puc/km }\end{array}$ & $\begin{array}{l}\text { Flow, } \\
\text { puc/h }\end{array}$ & $\begin{array}{l}\text { Speed, } \\
\mathrm{km} / \mathrm{h}\end{array}$ & $\begin{array}{l}\text { Density, } \\
\text { puc/km }\end{array}$ & $\begin{array}{l}\text { Flow, } \\
\text { puc/h }\end{array}$ & $\begin{array}{c}\text { Speed, } \\
\mathrm{km} / \mathrm{h}\end{array}$ & $\begin{array}{l}\text { Density, } \\
\text { puc/km }\end{array}$ & $\begin{array}{l}\text { Flow, } \\
\text { puc/h }\end{array}$ \\
\hline 1 & 57.72 & 84.79 & 3663.03 & 61.40 & 78.16 & 3739.40 & 60.78 & 79.40 & 3727.27 \\
\hline 2 & 58.53 & 82.27 & 3671.52 & 60.66 & 81.03 & 3921.21 & 59.12 & 83.03 & 3824.24 \\
\hline 3 & 58.80 & 81.62 & 3776.97 & 59.27 & 81.48 & 3767.27 & 53.15 & 91.32 & 3618.18 \\
\hline 4 & 56.45 & 80.60 & 3470.30 & 60.47 & 80.27 & 3778.18 & 54.49 & 91.38 & 3719.70 \\
\hline 5 & 56.00 & 86.97 & 3723.64 & 58.02 & 82.40 & 3699.40 & 58.53 & 82.24 & 3763.64 \\
\hline 6 & 56.52 & 86.73 & 3710.30 & 55.85 & 86.94 & 3621.82 & 57.33 & 84.19 & 3621.21 \\
\hline 7 & 58.21 & 85.56 & 3866.67 & 57.81 & 86.77 & 3827.88 & 56.16 & 87.74 & 3754.55 \\
\hline 8 & 57.833 & 84.95 & 3760.00 & 58.80 & 83.55 & 3766.06 & 55.73 & 87.44 & 3742.42 \\
\hline 9 & 56.47 & 86.40 & 3746.67 & 60.01 & 81.72 & 3803.64 & 57.83 & 84.91 & 3830.30 \\
\hline 10 & 54.23 & 89.15 & 3716.36 & 57.00 & 87.20 & 3795.15 & 55.65 & 88.61 & 3725.76 \\
\hline 11 & 40.06 & 113.61 & 3143.03 & 39.12 & 119.73 & 3204.85 & 33.12 & 134.98 & 2915.15 \\
\hline 12 & 20.28 & 179.20 & 2090.91 & 19.39 & 186.66 & 2009.70 & 11.68 & 237.86 & 1492.42 \\
\hline 13 & 12.96 & 224.13 & 1226.67 & 13.32 & 223.51 & 1208.49 & 5.26 & 309.43 & 369.70 \\
\hline 14 & 12.79 & 225.61 & 1130.91 & 12.70 & 225.57 & 1136.97 & 5.15 & 311.62 & 248.50 \\
\hline 15 & 12.68 & 225.28 & 1042.42 & 13.38 & 222.51 & 1111.52 & 5.14 & 311.43 & 269.70 \\
\hline 16 & 12.52 & 227.29 & 1099.39 & 13.48 & 223.49 & 1229.09 & 5.21 & 309.69 & 256.06 \\
\hline 17 & 12.46 & 227.36 & 1082.42 & 13.26 & 222.45 & 1216.97 & 5.22 & 309.18 & 259.09 \\
\hline 18 & 12.87 & 224.90 & 1060.61 & 13.04 & 222.01 & 1116.36 & 5.17 & 310.48 & 260.61 \\
\hline 19 & 12.98 & 222.42 & 1078.79 & 13.01 & 220.93 & 1105.45 & 5.10 & 311.27 & 266.67 \\
\hline 20 & 13.16 & 221.91 & 1255.76 & 13.13 & 222.40 & 1236.36 & 5.27 & 309.04 & 251.52 \\
\hline 21 & 13.22 & 221.50 & 1202.42 & 13.15 & 223.04 & 1139.40 & 5.13 & 310.51 & 230.30 \\
\hline 22 & 13.96 & 222.52 & 1277.58 & 12.92 & 224.99 & 1101.82 & 5.23 & 309.54 & 256.06 \\
\hline 23 & 13.09 & 223.99 & 1118.79 & 12.37 & 229.52 & 1073.94 & 4.99 & 313.61 & 225.76 \\
\hline 24 & 13.32 & 221.69 & 1089.70 & 12.35 & 228.94 & 1043.64 & 4.86 & 315.40 & 233.33 \\
\hline 25 & 12.61 & 226.18 & 1066.67 & 12.98 & 224.53 & 1067.88 & 5.09 & 311.69 & 231.82 \\
\hline 26 & 12.87 & 225.99 & 1174.55 & 13.26 & 221.53 & 1135.76 & 5.23 & 310.02 & 239.40 \\
\hline 27 & 12.48 & 226.84 & 1069.20 & 13.53 & 219.57 & 1180.61 & 5.14 & 310.00 & 268.18 \\
\hline 28 & 12.51 & 227.52 & 1044.85 & 13.65 & 218.93 & 1271.52 & 5.26 & 309.49 & 271.21 \\
\hline 29 & 12.46 & 226.93 & 1082.42 & 13.41 & 220.77 & 1160.00 & 5.25 & 309.72 & 271.21 \\
\hline 30 & 12.83 & 224.88 & 1126.06 & 12.71 & 226.21 & 1106.67 & 5.10 & 311.78 & 251.52 \\
\hline
\end{tabular}

TABLE 2: Calculation of system evolution parameters under occasional congestion.

\begin{tabular}{|c|c|c|c|}
\hline \multirow[b]{2}{*}{ Parameter } & \multicolumn{3}{|c|}{ Situation } \\
\hline & $\begin{array}{l}\text { Lane } 2 \text { is occupied } \\
\qquad \begin{aligned} a & =-0.3458 \\
b & =86.436\end{aligned}\end{array}$ & $\begin{array}{l}\text { Lane } 3 \text { is occupied } \\
\qquad \begin{aligned} a & =-0.5244 \\
b & =102.42\end{aligned}\end{array}$ & $\begin{array}{l}\text { Lanes } 2 \text { and } 3 \text { are occupied } \\
\text { at the same time } \begin{array}{l}a=-0.5655 \\
b=105.52\end{array}\end{array}$ \\
\hline Road capacity & 1863.08 & 2013.85 & 538.46 \\
\hline Traffic flow at breakpoints & 2433.14 & 2564.60 & 774.55 \\
\hline Change rate of the flow when it is stable & $-53.75 \%$ & $-56.01 \%$ & $-68.00 \%$ \\
\hline Change rate of capacity when it is stable & $21.45 \%$ & $22.82 \%$ & $30.52 \%$ \\
\hline
\end{tabular}

TABLE 3: Analysis of traffic flow control results under accidental accidents.

\begin{tabular}{lccc}
\hline Analysis of controlling efficiency & \multicolumn{2}{c}{$\begin{array}{c}\text { Situation } \\
\text { Lane 2 is occupied }\end{array}$} & $\begin{array}{c}\text { Lane 3 is occupied } \\
\text { Lanes 2 and 3 are occupied }\end{array}$ \\
\hline Flow restriction rate & $-70.29 \%$ & $-64.43 \%$ & $-91.44 \%$ \\
Road capacity without controlling & 1463.39 & 1554.32 & 374.12 \\
Road capacity with controlling & 1627.55 & 1776.22 & 394.75 \\
Efficiency of flow controlling & $11.22 \%$ & $14.28 \%$ & $5.51 \%$ \\
\hline
\end{tabular}

3 is occupied. Analyzing the reasons, the remaining capacity of the road when one lane is occupied is greater than when two lanes are occupied, and the remaining capacity of the road when lane 3 is occupied is greater than when lane 2 is occupied. As a result, it can be obtained that the traffic flow controlling method based on the catastrophe characteristics 
is more effective when the accidental accident has a small impact on the road capacity, which can better control the congestion and improve the road capacity.

\section{Conclusion}

In this paper, taking the occupancy rate of the traffic volume in the carrying capacity of the road system as the state variable, the change rate of the traffic flow, and the change rate road capacity as the control variables, a cusp catastrophe model of the road traffic system is constructed under occasional congestion. Find breakpoints and analyze stability after urban road system catastrophes by constructing the energetic function; based on the catastrophe characteristics of the urban road system, the feasibility method of congestion control is discussed. And give the traffic flow control method on this basis: (1) Before a catastrophe, control the traffic flow to make it less than the breakpoints. Preventing the catastrophe occurs, and the potential function decreases sharply. (2) After a catastrophe, control the traffic flow with a certain restriction rate to be as small as the stable traffic flow, preventing the potential function from moving to a smaller direction, in order to obtain the maximum capacity. Through example analysis, it is found that, in the case of accidental accidents that have less impact on road capacity, the control method of traffic flow based on the catastrophe characteristics is more effective and can better control congestion and improve road capacity; in the case of accidental accidents, the traffic flow control method is more effective. When the road capacity is greatly affected, it is difficult to effectively improve the capacity of the urban road system only by controlling the traffic flow.

\section{Data Availability}

The data were obtained from experiments of the traffic flow simulation procedures.

\section{Conflicts of Interest}

The authors declare that they have no conflicts of interest.

\section{Acknowledgments}

This study was supported by the National Social Science Fund of China (19BGL275) and Department of Science and Technology Project of Shaanxi Province (2020JQ-654).

\section{References}

[1] G. Hong Cheng, S. Li-Jun, and J.-Y. Chen, "Modeling and control of urban expressway traffic flow," Journal of Highway and Transportation Research and Development, vol. 23, no. 8, pp. 101-105, 2006.

[2] J. Guo, X. L. Chen, and H. Z. Jin, "Research on model of traffic flow based on cusp catastrophe," Control and Decision, vol. 2, pp. 237-240, 2008.

[3] C. Tao and C. Senfa, "Research on models of Congestion control based on catastrophe theory," Journal of Systems Engineering, vol. 21, no. 6, pp. 598-605, 2007.
[4] J. C. Long, Z. Y. Gao, and H. L. Ren, "Dynamic signal control method of urban network traffic," China Journal of Highway and Transport, vol. 22, no. 4, pp. 108-114, 2009.

[5] R. Thom, Structural Stabiltiy and Morphogenesis, W. A. Benjamin, Inc, vol. 36, no. 5-6, , pp. 611-613, San Francisco, CA, USA, 1972.

[6] L. Fuhua, Catastrophe Theory and its Application, pp. 1-6, Shanghai Jiao Tong University Press, Shanghai, China, 1987.

[7] L. Jiqi, "Study on waterproof and drainage of arch tunnel," Shanxi Architecture, vol. 3, pp. 299-301, 2007.

[8] J. Gu and S. Chen, "Nonlinear analysis on traffic flow based on catastrophe and chaos theory discrete dynamics in nature and society," Discrete Dynamics in Nature and Society, vol. 2014, Article ID 535167, 11 pages, 2014.

[9] L. Xu, L. Li, L. Guang-Ze et al., "Review of multilane traffic flow theory and application," Journal of Chang'an University: Natural Science Edition, vol. 40, no. 4, pp. 78-90, 2020.

[10] A. E. Papacharalampous and E. I. Vlahogianni, "Modeling microscopic freeway traffic using cusp catastrophe theory," IEEE Intelligent Transportation Systems Magazine, vol. 6, no. 1, pp. 6-16, 2014.

[11] W. Dianhai, Traffic Flow Theory, China Communications Press, Beijing, China, 2002.

[12] H. B. Zhu, H. X. Ge, L. Y. Dong, and S. Q. Dai, “A modified $\mathrm{NaSch}$ model with density-dependent randomization for traffic flow," The European Physical Journal B, vol. 57, no. 1, pp. 103-108, 2007.

[13] Y. Xu, D. Zhang, and A. J. K. Chowdhury, "Urban road traffic flow control under incidental congestion as a function of accident duration," Open Physics, vol. 16, no. 1, pp. 1085-1093, 2018 . 